\title{
Automated Surface Matching Using Mutual Information Applied to Riemann Surface Structures
}

\author{
Yalin Wang ${ }^{1}$, Ming-Chang Chiang ${ }^{2}$, and Paul M. Thompson ${ }^{2}$ \\ 1 Mathematics Department, UCLA, Los Angeles, CA 90095, USA \\ 2 Lab. of Neuro Imaging, UCLA School of Medicine, Los Angeles, CA 90095, USA \\ ylwang@math.ucla.edu, mcchiang@ucla.edu, thompson@loni.ucla.edu
}

\begin{abstract}
Many medical imaging applications require the computation of dense correspondence vector fields that match one surface with another. To avoid the need for a large set of manually-defined landmarks to constrain these surface correspondences, we developed an algorithm to automate the matching of surface features. It extends the mutual information method to automatically match general 3D surfaces (including surfaces with a branching topology). First, we use holomorphic 1-forms to induce consistent conformal grids on both surfaces. High genus surfaces are mapped to a set of rectangles in the Euclidean plane, and closed genus-zero surfaces are mapped to the sphere. Mutual information is used as a cost functional to drive a fluid flow in the parameter domain that optimally aligns stable geometric features (mean curvature and the conformal factor) in the $2 \mathrm{D}$ parameter domains. A diffeomorphic surfaceto-surface mapping is then recovered that matches anatomy in $3 \mathrm{D}$. We also present a spectral method that ensures that the grids induced on the target surface remain conformal when pulled through the correspondence field. Using the chain rule, we express the gradient of the mutual information between surfaces in the conformal basis of the source surface. This finite-dimensional linear space generates all conformal reparameterizations of the surface. We apply the method to hippocampal surface registration, a key step in subcortical shape analysis in Alzheimer's disease and schizophrenia.
\end{abstract}

\section{Introduction}

In computational anatomy, surface-based computations are used to statistically combine or compare 3D anatomical models across subjects, or map functional imaging parameters onto anatomical surfaces. When comparing data on two anatomical surfaces, a correspondence field must be computed to register one surface nonlinearly onto the other. Multiple surfaces can be registered nonlinearly to construct a mean shape for a group of subjects, and deformation mappings can encode shape variations around the mean. This type of deformable surface registration has been used to detect developmental and disease effects on 
brain structures such as the corpus callosum and basal ganglia [1, the hippocampus [2], and the cortex [3]. Nonlinear matching of brain surfaces can also be used to track the progression of neurodegenerative disorders such as Alzheimer's disease [2, to measure brain growth in development [1, and to reveal directional biases in gyral pattern variability [4].

Surface registration has numerous applications, but a direct mapping between two 3D surfaces is challenging to compute. Often, higher order correspondences must be enforced between specific anatomical points, curved landmarks, or subregions lying within the two surfaces. This is often achieved by first mapping each of the 3D surfaces to canonical parameter spaces such as a sphere [56] or a planar domain 7]. A flow, computed in the parameter space of the two surfaces [18, then induces a correspondence field in 3D. This flow can be constrained using anatomic landmark points or curves, or by constraining the mapping of surface regions represented implicitly using level sets [7]. Feature correspondence between two surfaces can be optimized by using the $L^{2}$-norm to measure differences in convexity [5]. Artificial neural networks can rule out or favor certain types of feature matches [9. Finally, correspondences may be determined by using a minimum description length (MDL) principle, based on the compactness of the covariance of the resulting shape model [10]. Anatomically homologous points can then be forced to match across a dataset. Thodberg [11] identified problems with early MDL approaches and extended them to an MDL appearance model, when performing unsupervised image segmentation.

By the Riemann uniformization theorem, all surfaces can be conformally embedded in a sphere, a plane or a hyperbolic space. The resulting embeddings form special groups. Using holomorphic 1-forms and critical graphs, global conformal parameterization [12] can be used to conformally map any high genus surface (i.e., a surface with branching topology) to a set of rectangular domains in the Euclidean plane. In this paper, we use conformal parameterizations to help match arbitrary 3D anatomical surfaces. Mutual information is used to drive a diffeomorphic fluid flow that is adjusted to find appropriate surface correspondences in the parameter domain. We chose the mean curvature and the conformal factor of the surfaces as the differential geometric features to be aligned in this study as they are intrinsic and stable. These choices are illustrative - any scalar fields defined on the surfaces could be matched, e.g. cortical thickness maps, functional imaging signals or metabolic data. Since conformal mapping and fluid registration techniques generate diffeomorphic mappings, the 3D shape correspondence established by composing these mappings is also diffeomorphic (i.e., provides smooth one-to-one correspondences).

\section{Theoretical Background and Definitions}

Due to space limitations, here we list some formal definitions that help describe our approach, without detailed explanation. For further reading, please refer to [13] and [14. 


\subsection{Surface Parameterization with Riemann Surface Structure}

An atlas is a collection of consistent coordinate charts on a manifold, where transition functions between overlapping coordinate charts are smooth. We treat $R^{2}$ as isomorphic to the complex plane. Let $S$ be a surface in $\mathbb{R}^{3}$ with an atlas $\left\{\left(U_{\alpha}, z_{\alpha}\right)\right\}$, where $\left(U_{\alpha}, z_{\alpha}\right)$ is a chart, and $z_{\alpha}: U_{\alpha} \rightarrow \mathbb{C}$ maps an open set $U_{\alpha} \subset S$ to the complex plane $\mathbb{C}$. An atlas is called conformal if (1). each chart $\left(U_{\alpha}, z_{\alpha}\right)$ is a conformal chart. Namely, on each chart, the first fundamental form can be formulated as $d s^{2}=\lambda\left(z_{\alpha}\right)^{2} d z_{\alpha} d \overline{z_{\alpha}} ;$ (2). the transition maps $z_{\beta} \circ z_{\alpha}^{-1}$ : $z_{\alpha}\left(U_{\alpha} \cap U_{\beta}\right) \rightarrow z_{\beta}\left(U_{\alpha} \cap U_{\beta}\right)$ are holomorphic.

A chart is compatible with a given conformal atlas if adding it to the atlas again yields a conformal atlas. A conformal structure (Riemann surface structure) is obtained by adding all compatible charts to a conformal atlas. A Riemann surface is a surface with a conformal structure. It has been proven that all metric orientable surfaces are Riemann Surfaces.

One coordinate chart in the conformal structure introduces a conformal parameterization between a surface patch and the image plane. The conformal parameterization is angle-preserving and intrinsic to the geometry.

The surface conformal structure induces special curvilinear coordinate system on the surfaces. Based on a global conformal structure, a critical graph can be recovered that connects zero points in the conformal structure and partitions a surface into patches. Each of these patches can be conformally mapped to a parallelogram by integrating a holomorphic 1-form defined on the surface. Figure 1(a)-(c) show an example of the conformal parameterization of a lateral ventricle surface of a 65 -year-old HIV/AIDS patient. The conformal structure of

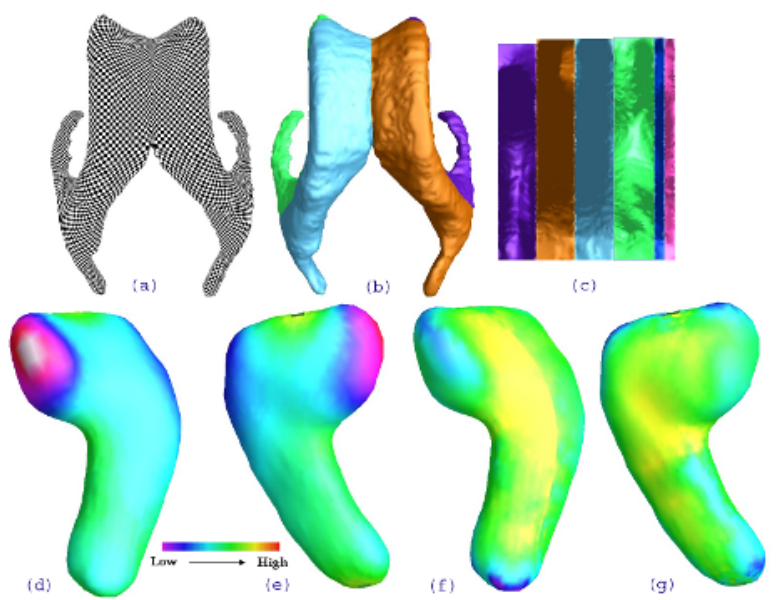

Fig. 1. Illustrates conformal surface parameterization. (a) - (c) illustrate conformal parameterizations of ventricular surfaces in the brain for a 65-year-old HIV/AIDS patient. (d)-(g) show the computed conformal factor and mean curvature on a hippocampal surface, (d)-(e) are two views of the hippocampal surface, colored according to conformal factor; (f)-(g) are two views of the hippocampal surface, colored according to mean curvature. 
the ventricular surface is shown in (a). (b) shows a partition of the ventricular surface, where each segment is labeled by a unique color. (c) shows the parameterization domain, where each rectangle is the image, in the parameterization domain, of a surface component in (b).

For a Riemann surface $S$ with genus $g>0$, all holomorphic 1-forms on $S$ form a complex $g$-dimensional vector space ( $2 g$ real dimensions), denoted by $\Omega^{1}(S)$. The conformal structure of a higher genus surface can always be represented in terms of a holomorphic one-form basis, which is a set of $2 g$ functions $\omega_{i}: K_{1} \rightarrow$ $R^{2}, i=1,2 \cdots, 2 g$. Any holomorphic one-form $\omega$ is a linear combination of these functions. The quality of a global conformal parameterization for a high genus surface is fundamentally determined by the choice of the holomorphic 1-form.

\subsection{Conformal Representation of a General Surface}

For a general surface $S$, we can compute conformal coordinates $(u, v)$ to parameterize $S$. Based on these coordinates, one can derive scalar fields including the conformal factor, $\lambda(u, v)$, and mean curvature, $H(u, v)$, of the surface position vector $S(u, v): \frac{\partial S}{\partial u} \times \frac{\partial S}{\partial v}=\lambda(u, v) n(u, v)$, and $H(u, v)=1 \frac{1}{\lambda^{2}(u, v)}\left(\frac{\partial^{2}}{\partial u^{2}}+\right.$ $\left.\frac{\partial^{2}}{\partial v^{2}}\right) r(u, v) \mid$. From the theorem [15], we can regard the tuple $(\lambda, H)$ as the conformal representation of $S(u, v)$.

Clearly, various fields of scalars or tuples could be used to represent surfaces in the parameter domain. Because the conformal structure is intrinsic and independent of the data resolution and triangulation, we use the conformal representation, $\lambda(u, v)$ and $H(u, v)$, to represent the $3 \mathrm{D}$ surfaces. This representation is stable and computationally efficient. Figure 1 (d)-(g) shows the conformal factor $((\mathrm{d})$ and $(\mathrm{e}))$, and mean curvature ((f) and (g)), indexed in color on a hippocampal surface.

\subsection{Mutual Information (MI) for Surface Registration}

We now describe the mutual information functional used to drive the scalar fields $\lambda(u, v)$ and $H(u, v)$ into correspondence, effectively using the equivalent of a $2 \mathrm{D}$ image registration in the surface parameter space (i.e., in conformal coordinates). Let $I_{1}$ and $I_{2}$ be the target and the deforming template images respectively, and $I_{1}, I_{2}: R^{2} \rightarrow R$. Let $\Omega \subset R^{2}$ be the common parameter domain of both surfaces (if both are rectangular, the target parameter domain is first matched to the source parameter domain using a $2 \mathrm{D}$ diagonal matrix). Also, let $u$ be a deformation vector field on $\Omega$. The MI of the scalar fields (treated as $2 \mathrm{D}$ images) between the two surfaces is defined by $I(u)=\int_{R^{2}} p_{u}\left(i_{1}, i_{2}\right) \log \frac{p_{u}\left(i_{1}, i_{2}\right)}{p\left(i_{1}\right) p_{u}\left(i_{2}\right)} d i_{1} d i_{2}$. where $p\left(i_{1}\right)=P\left(I_{1}(x)=i_{1}\right), p_{u}\left(i_{2}\right)=P\left(I_{2}(x-u)=i_{2}\right)$ and $p_{u}\left(i_{1}, i_{2}\right)=$ $P\left(I_{1}(x)\right)=i_{1}$ and $I_{2}(x-u)=i_{2}$.

We adopted the framework of D'Agostino et al. 16 to maximize MI with viscous fluid regularization. Briefly, the deforming template image was treated as embedded in a compressible viscous fluid governed by Navier-Stokes equation for conservation of momentum [17, simplified to a linear PDE:

$$
L v=\mu \nabla^{2} v+(\lambda+\mu) \nabla(\nabla \cdot v)+F(x, u)=0
$$


Here $v$ is the deformation velocity, and $\mu$ and $\lambda$ are the viscosity constants. Following [16], we take the first variation of $I(u)$ with respect to $u$, and use the Parzen window method [18 to estimate the joint probability density function (pdf) $p_{u}\left(i_{1}, i_{2}\right)$.

\section{The Surface Mutual Information Method for an Arbitrary Genus Surface}

To match two high genus surfaces (i.e., surfaces with the same branching topology), we apply our surface mutual information method piecewise. First, we compute conformal representations of the two surfaces based on a global conformal parameterization. 11 These conformal representations are aligned with mutual information driven flows, while enforcing constraints to guarantee that the vectorvalued flow is continuous at the patch boundaries. 2 When the chain rule is used, we can further optimize the mutual information matching results by optimizing the underlying global conformal parameterization.

Let $S_{1}$ and $S_{2}$ be two surfaces we want to match and the conformal parameterization of $S_{1}$ is $\tau_{1}$, conformal parameterization for $S_{2}$ is $\tau_{2}, \tau_{1}\left(S_{1}\right)$ and $\tau_{2}\left(S_{2}\right)$ are rectangles in $R^{2}$. Instead of finding the mapping $\phi$ from $S_{1}$ to $S_{2}$ directly, we can use mutual information method to find a diffeomorphism $\tau: D_{1} \rightarrow D_{2}$, such that: $\tau_{2}^{-1} \circ \tau \circ \tau_{1}=\phi$. Then the map $\phi$ can be obtained from $\phi=\tau_{1} \circ \tau \circ \tau_{2}^{-1}$. Because $\tau_{1}, \tau$ and $\tau_{2}$ are all diffeomorphisms, $\phi$ is also a diffeomorphism.

\subsection{Mutual Information Contained in Maps Between High Genus Surfaces}

A global conformal parameterization for a high genus surface can be obtained by integrating a holomorphic one-form $\omega$. Suppose $\left\{\omega_{i}, i=1,2, \cdots, 2 g\right\}$ is a holomorphic 1-form basis, where an arbitrary holomorphic 1-form has the formula

1 Several variational or PDE-based methods have been proposed to match surfaces represented by parametric meshes [5], level sets, or both [7]. Gu et al. [19] found a unique conformal mapping between any two genus zero manifolds by minimizing the harmonic energy of the map. Gu and Vemuri 20] also matched genus-zero closed 3D shapes by first conformally mapping them to a canonical domain and aligning their $2 \mathrm{D}$ representations over the class of diffeomorphisms.

2 The mutual information method 14 measures the statistical dependence of the voxel intensities between two images. Parameters of a registration transform can be tuned so that MI is maximal when the two images are optimally aligned. The MI method has been successful for rigid [21] and non-rigid [22 23] image registration. Here, we generalize it to match $3 \mathrm{D}$ surfaces. For MI to work, a monotonic mapping in grayscales between images is not required, so images from different modalities can be registered [24. Hermosillo et al. 25] adopted linear elasticity theory to regularize the variational maximization of MI. D'Agostino et al. [16] extended this approach to a viscous fluid scheme allowing large local deformations, while maintaining smooth, one-to-one topology [17. 
$\omega=\sum_{i=1}^{2 g} \lambda_{i} \omega_{i}$. Assuming the target surface's parameterization is fixed, the mutual information between it and the source surface's parameterization is denoted $E(\omega)$, which is a function of the linear combination of coefficients $\lambda_{i}$. A necessary condition for the optimal holomorphic 1 -form is, $\frac{\partial E}{\partial \lambda_{i}}=0, i=1,2, \cdots, 2 g$. If the Hessian matrix $\left(\frac{\partial^{2} E}{\partial \lambda_{i} \partial \lambda_{j}}\right)$ is positive definite, then $E$ will reach the minimum. If the Hessian matrix is negative definite, $E$ will be maximized.

Our surface mutual information method depends on the selection of holomorphic 1-form $\omega$. To match surfaces optimally, we find the holomorphic 1-form that maximizes the mutual information metric. Suppose a holomorphic function $\omega=\sum_{i=1}^{2 g} \lambda_{i} \omega_{i}$, our goal is to find a set of coefficients $\lambda_{i}, i=1, \ldots, 2 g$ that maximize the mutual information, $E_{M I}$. We solve this optimization problem numerically as follows:

$$
d E_{M I}=\left(\frac{d E_{M I}}{d u}, \frac{d E_{M I}}{d v}\right)\left(\begin{array}{cccc}
\frac{d u}{d \lambda_{1}} & \frac{d u}{d \lambda_{2}} & \ldots & \frac{d u}{d \lambda_{2 g}} \\
\frac{d v}{d \lambda_{1}} & \frac{d v}{d \lambda_{2}} & \ldots & \frac{d v}{d \lambda_{2 g}}
\end{array}\right)\left(\begin{array}{c}
d \lambda_{1} \\
d \lambda_{2} \\
\ldots \\
d \lambda_{2 g}
\end{array}\right)
$$

where $(u, v)$ is the conformal coordinate.

Once we compute $\frac{d E_{M I}}{d \lambda_{i}}, i=1,2, \ldots, 2 g$, we can use steepest descent to optimize the resulting mutual information. A complete description of the surface mutual information method follows.

Algorithm 1. Surface MI Method (for surfaces of arbitrary genus) Input (mesh $M^{1}, M^{2}$,step length $\delta t, M I$ difference threshold $\delta E$ ), Output $\left(t: M^{1} \rightarrow M^{2}\right)$ where $t$ minimizes the surface mutual information.

1. Compute global conformal parameterization of two surfaces, $\omega^{j}=\sum_{i=1}^{2 g} s_{i} \omega_{i}$, $j=1,2 ; i=1,2, \ldots, 2 g$, where $g$ is the surface genus number of two surfaces $M^{1}$ and $M^{2}$, and $s_{i}^{j}, j=1,2, i=1,2, \ldots, 2 g$ are the coefficients of a linear combination of holomorphic function basis elements. The steps include computing the homology basis, cohomology basis, harmonic one-form basis and holomorphic one-form basis.

2. Compute holomorphic flow segmentation of the target surface, $M^{2}$, from the global conformal parameterization, $\omega^{2}$, which conformally maps the $3 D$ surface to a set of rectangles in the Euclidean plane.

3. Compute $2 D$ conformal representation for the target surface, $\lambda^{2}(u, v)$ and $H^{2}(u, v)$, where $(u, v)$ is the conformal coordinate;

4. Compute holomorphic flow segmentation of the source surface, $M^{1}$, and $2 D$ conformal representation $\lambda^{1}(u, v)$ and $H^{1}(u, v)$;

5. Apply the mutual information method to optimize the correspondence between two surfaces, $t:\left(\lambda^{1}(u, v), H^{1}(u, v)\right) \rightarrow\left(\lambda^{2}(u, v), H^{2}(u, v)\right), j=1,2$ and $H^{j}(u, v), j=1,2$; and compute mutual information $E_{M I}^{0}$;

6. Compute derivative Dt.

7. Update the global conformal parameterization of source surface, $M^{1}$, by changing the coefficients $s^{1}(v)=D t(v) \delta t$.

8. Compute mutual information E, with steps $3,4,5$.

9. If $E_{M I}-E_{M I}^{0}<\delta E$, return $t$. Otherwise, assign $E$ to $E_{0}$ and repeat steps 6 through 9 . 
Currently, we use the following numerical scheme in step 6:

1. Compute $d E_{M I} / d u$ and $d E_{M I} / d v, d u / d s_{i}, i=1,2, \ldots, 2 g$;

2. Compute $d v / d s_{i}, i=1,2, \ldots, 2 g$;

3. Compute $D t=d E_{M I} / d s_{i}, i=1,2, \ldots, 2 g$ with Equation 2.

\section{Experimental Results}

To make the results easier to illustrate, we chose to encode the profile of surface features using a compound scalar function $C(u, v)=8 \lambda(u, v)+H(u, v)$, where $\lambda(u, v)$ is conformal factor and $H(u, v)$ the mean curvature. Several examples are shown matching hippocampal surfaces in 3D. This type of deformable surface registration can help track developmental and degenerative changes in the brain, and can create average shape models with appropriate boundary correspondences. In the experiments, the velocity field $v$ in Equation 1 was computed iteratively by convolution of the force field with a filter kernel derived by BroNielsen and Gramkow [26]. The viscosity coefficients $\lambda$ and $\mu$ were set to 0.9 and 6.0 respectively. The deformation field in the parameter domain $(u)$ was obtained from $v$ by Euler integration over time, and the deformed template image was regridded when the Jacobian determinant of the deformation mapping at any point in $x-u$ was smaller than 0.5 [17. At each step, the joint pdf was updated and the MI re-computed. Iterations were stopped when MI was no longer monotonically increasing or when the number of iterations reached 350 . The Parzen parameter $h$ was set to 10 for smoothing the joint pdf. Figure 2 (a) shows the matching fields for several pairs of surfaces, establishing correspondences between distinctive features. Here geometric features on 3D hippocampal surface, conformal factor and mean curvature, were conformally flattened to a $2 \mathrm{D}$ square. In the 2D parameter domain, data from a healthy control subject was registered to data from several Alzheimer's disease patients. Each mapping can be used to obtain a reparameterization of the 3D surface of the control subject, by convecting the original 3D coordinates along with the flow. Importantly, in Figure 2 (a), some consistent 3D geometric features are identifiable in the 2D parameter domain; bright area (arrows) correspond to high curvature features in the hippocampal head.

Although validation on a larger sample is required, we illustrate the approach on left hippocampal surface data from one healthy control subject and five patients with Alzheimer's disease. We register the control subject's surface to each patient, generating a set of deformation mappings. Figure 2 (b)-(e) show the correspondences as a $3 \mathrm{D}$ vector field map connecting corresponding points on two surfaces being registered. (d) and (e) are a part of surface before and after the registration. After reparameterization, a leftward shift in the vertical isocurves adds a larger tangential component to the vector field. Even so, the deformed grid structure remains close to conformal. The lengths of the difference vectors are reduced after the MI based alignment; a formal validation study in a larger sample is now underway. 


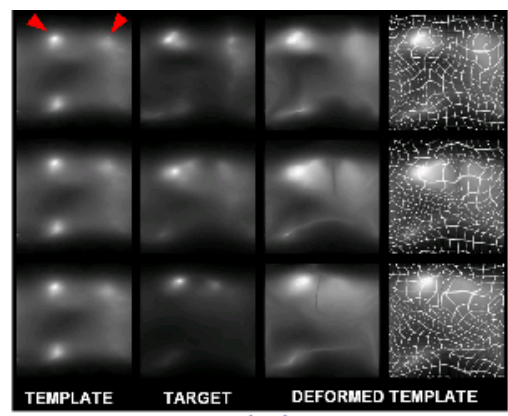

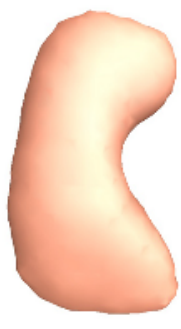

(b)

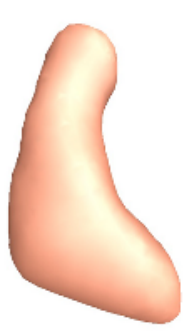

(c)

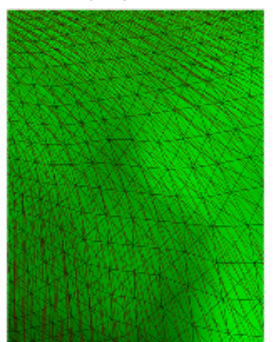

(d)

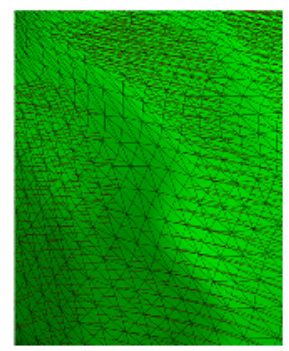

(e)

Fig. 2. (a) Geometric features on 3D hippocampal surfaces (the conformal factor and mean curvature) were computed and compound scalar fields were conformally flattened to a $2 \mathrm{D}$ square. In the $2 \mathrm{D}$ parameter domain, data from a healthy control subject (the template, leftmost column) was registered to data from several Alzheimer's disease patients (target images, second column). The deformed template images are shown in the third and fourth (gridded) columns. (b)-(c) show the two 3D hippocampal surfaces being matched, for (b) a control subject and (c) an Alzheimer's disease patient. We flow the surface from (b) to (c). (d)-(e) show the $3 \mathrm{D}$ vector displacement map, connecting corresponding points on the two surfaces, (d) before and (e) after reparameterization of the source surface using a fluid flow in the parameter domain. These $3 \mathrm{D}$ vector fields store information on geometrical feature correspondences between the surfaces.

\section{Conclusions and Future Work}

We extended the mutual information method to match general surfaces. This has many applications in medical imaging. Future work will validate the matching of hippocampal surfaces in shape analysis applications in degenerative diseases, as well as building statistical shape models to detect the anatomical effects of disease, aging, and development. The hippocampus is used as a specific example, but the method is general and is applicable in principle to other brain surfaces such as the cortex. Whether or not our new method provides a more relevant correspondences than those afforded by other criteria (minimum description length, neural nets, or hand landmarking) requires careful validation for each application. Because different correspondence principles produce different shape models, we plan to compare them in future for detecting group differences in brain structure. 


\section{References}

1. Thompson, P., et al. Nature 404 (2000) 190-193

2. Csernansky, J., et al. Proc. Natl. Acad. Sci. 95 (1998) 11406-11411

3. Thompson, P., et al. In: Human Brain Mapping. Volume 9. (2000) 81-92

4. Kindlmann, G., et al. In: Proc. IEEE EMBS, San Francisco, CA (2004)

5. Fischl, B., et al. In: Human Brain Mapping. Volume 8. (1999) 272-284

6. Bakircioglu, M., et al. In: SPIE Medical Imaging. Volume 3661. (1999) 710-715

7. Leow, A., et al. NeuroImage 24 (2005) 910-927

8. Davatzikos, C. J. Comp. Assisted Tomography 20 (1996) 656-665

9. Pitiot, A., et al. In: IPMI. (2003) 25-37

10. Davis, R., et al. In: IEEE TMI. Volume 21. (2002) 525-537

11. Thodberg, H. In: IPMI. (2003) 51-62

12. Gu, X., Yau, S. Communication of Information and Systems 2 (2002) 121-146

13. Schoen, R., Yau, S.T.: Lectures on Harmonic Maps. International Press (1997)

14. Wells, W., et al. Medical Image Analysis 1 (1996) 35-51

15. Gu, X., et al. Communication of Information and Systems 3 (2005) 171-182

16. D'Agostino, E., et al. Medical Image Analysis 7 (2003) 565-575

17. Christensen, G., et al. IEEE TIP 5 (1996) 1435-1447

18. Parzen, E. The annals of mathematical statistics 33 (1962) 1065-1076

19. Gu, X., et al. IEEE TMI 23 (2004) 949-958

20. Gu, X., Vemuri, B. In: MICCAI. (2004) 771-780

21. West, J., et al. Journal of Computer Assisted Tomography 21 (1997) 554-566

22. Meyer, C., et al. Medical Image Analysis 1 (1997) 195-206

23. Rueckert, D., et al. IEEE TMI 18 (1999) 712-721

24. Kim, B., et al. NeuroImage 5 (1997) 31-40

25. Hermosillo, G. PhD thesis, Universitè de Nice (INRIA-ROBOTVIS), Sophia Antipolis, France (2002)

26. Bro-Nielsen, M., Gramkow, C. In: Visualization in Biomedical Computing (VBC'96). Volume 1131., Springer (1996) 267-276 